\title{
L'évolution des termes d'adresse à contenu social en ancien et moyen français
}

\author{
Sabine LEHMANN \\ Université Paris Ouest Nanterre La Défense \\ Laboratoire MoDyCo \\ sabine_lehman@yahoo.fr
}

Recibido: 1 de noviembre de 2010

Aceptado: 18 de diciembre de 2010

\begin{abstract}
RÉSUMÉ
A partir d'un corpus de textes en ancien et moyen français, on analyse le fonctionnement des groupes nominaux vocatifs dans le discours direct. Après une analyse des définitions courantes des notions de vocatif, apostrophe et appellatif, nous présentons la notion de terme d'adresse (groupe nominal détaché et employé vocativement dans le discours direct pour interpeller l'allocutaire), comme opposée à la notion de désignatif (groupe nominal strictement référentiel renvoyant aux locuteur, allocutaire ou délocuté). Nous étudions les termes d'adresse du point de vue sociolinguistique, en tant que marqueurs de l'évolution des statuts des locuteurs, marqueurs des rapports de places et des changements de sphères. La nature grammaticale des termes d'adresse est définie et comparée avec celle des désignatifs. On passe ensuite à l'étude des positions des termes d'adresse dans les énoncés du corpus. La validité du principe fréquemment soutenu selon lequel le terme d'adresse a essentiellement une fonction de marqueur de début de discours est testée.
\end{abstract}

Mots clés: Terme d'adresse, discours direct, statut social, localisation du discours.

\section{La evolución de los términos de apelación en francés antiguo y medio}

\section{RESUMEN}

A partir de un corpus textual en francés antiguo y medio, se analiza el funcionamiento de los grupos nominales vocativos en el discurso directo. Tras un análisis de las definiciones habituales de las nociones de vocativo, apóstrofe y apelativo, presentamos la noción de término de apelación (grupo nominal aislado y empleado vocativamente en el discurso directo para interpelar al alocutario), como opuesto a la noción de designativo (sintagma nominal estrictamente referencial que reenvía al locutor, alocutario o delocutado). Estudiamos los términos de apelación desde el punto de vista sociolingüístico, como marcadores de la evolución del estatus de los locutores, marcadores de las relaciones entre posiciones y de los cambios de ámbito. La naturaleza gramatical de los términos de apelación se define y compara con la de los designativos. Inmediatamente se pasa al estudio de las posiciones de los términos de apelación en los enunciados del corpus. Queda así comprobada la validez del principio afirmado frecuentemente de que el término de apelación tiene una función de marcador de comienzo del discurso.

Palabras clave: Término de apelación, estilo directo, posición social, localización del discurso. 


\title{
The evolution of terms of address with social contents in old and middle French
}

\begin{abstract}
Based on a corpus of texts in Old and Middle French, this research paper examines the behavior of vocative nominal groups in direct speech. After analyzing the currently available descriptions of vocative, apostrophe and appellative, we present the concept "terms of address" (detached nominal groups used vocatively in direct speech to address the co-locutor, i.e. the addressee), in contrast with "designatives" (bound nominal groups used in narrative to refer to the locutor, the addressee or the third person-délocuté). We adopt a sociolinguistic approach by which terms of address are markers of social status, place and sphere. Before presenting an analysis of the position of terms of address within utterances from the corpus, we define the grammatical nature of terms of address, and then compare it to the nature of designatives. The generally accepted proposition that terms of address are mainly speech markers is brought under scrutiny and put into question.
\end{abstract}

Key words: Terms of address, direct speech, social status, location of direct speech.

Sommaire : 1 - Du désignatif au terme d'adresse : mise au point terminologique et sociolinguistique. - 2 - Du vers à la prose : le destin des termes d'adresse. 3 - Analyse du corpus : 3.1 - Le monde en vers et en prose. 3.2 - La localisation du discours.

Notre étude est consacrée à l'évolution des syntagmes nominaux détachés sans fonction dans la proposition, appelés termes d'adresse (Lagorgette, 1998), dans un corpus représentatif des périodes de l'ancien et du moyen français. En effet, les termes d'adresse (dits aussi syntagmes vocatifs, appellatifs, apostrophes, selon les écoles grammaticales ou linguistiques) existent dans toutes les langues vivantes et mortes. Nous nous intéresserons en particulier aux éléments constitutifs du vocabulaire de la stratification sociale fonctionnant comme termes d'adresse, afin de pouvoir établir des parallèles entre l'évolution des structures sociales et leur reflet dans les différents univers littéraires qui caractérisent la période médiévale (chansons de geste, romans du cycle «Lancelot-Graal», poésie allégorique et amoureuse de Charles d'Orléans, poésie du quotidien de Rutebeuf ou Eustache Deschamps, les Chroniques de Froissart ainsi que les textes sur la condition féminine de Christine de Pisan).

Après une mise au point terminologique concernant la différence à établir entre les désignatifs et les termes d'adresse, nous montrerons selon quels systèmes s'inscrit la parole dans un texte qui fut écrit en vers, puis mis en prose. Parmi les indices qui servent à indiquer la présence de la parole, c'est l'utilisation de marques lexicales sous formes de termes d'adresse que nous allons mettre au premier plan de notre étude. Le phénomène de mise en prose nous servira de point de départ pour une comparaison plus vaste concernant le fonctionnement des termes d'adresse dans des textes rédigés en vers, d'une part et dans des productions littéraires en prose, d'autre part. Les termes d'adresse seront étudiés dans le cadre de la prise en considération des réalités sociales. Il s'agit d'établir un lien entre la prédominance d'un type social intégrant un texte sous forme d'un terme d'adresse, et la nature du texte ainsi que le contexte social qui a conditionné l'acte d'écriture. Nous nous 
interrogerons en même temps sur le rapport entre la prédominance de telle ou telle catégorie sociale dans les textes et sa place réelle dans la société médiévale.

\section{Du désignatif au terme d'adresse : mise au point terminologique et sociolinguistique.}

Tandis que le mot adresse renvoie à la fonction appellative, dans la terminologie traditionnelle ${ }^{1}$, le mot désignatif est clairement du côté de la mention. En effet, il s'agit de deux classes différentes de groupes nominaux de dénomination. Pour V. Alleton (1981), il est évident que ces groupes nominaux ne remplissent pas les mêmes fonctions :

On distingue le terme d'adresse (on parle à autrui) de la désignation (on parle d'autrui à des tiers) - la 'présentation' étant un cas particulier de la désignation. L'adresse et la désignation peuvent être formellement distinctes ou non. En français, les noms propres et certaines appellations de parenté occasionnellement employées comme désignation peuvent avoir la même forme: 'Michel', 'Dupont', 'Papa', mais la plupart des termes diffèrent par leurs déterminations : Monsieur / le monsieur ; Votre Excellence / Son Excellence ; camarades ! / mes (les) camarades, etc. (...) [La fonction adresse] ne se conçoit que dans le discours; elle est exclue du récit. (Alleton, $1981: 46-47$ )

Comme Alleton, de nombreux auteurs ${ }^{2}$ soulignent que les termes d'adresse sont exclusivement du côté du discours et s'opposent donc aux syntagmes nominaux désignatifs qui font partie du réseau de référence.

Tandis que les spécialistes de la philosophie du langage (Gottlob Frege, 1952 ; Saul Kripke, 1980 ; et plus récemment F. Récanati, 1979; G. Kleiber, 1981 ; M.-N. Gary-Prieur, 1994 et Francis Corblin, 1995), opèrent avec la notion de désignateur qui renvoie à différentes sous-catégories nécessitant des précisions (désignateur rigide / désignateur non-rigide) dans le domaine auquel elles appartiennent, le terme de désignatif s'installe dans des travaux de recherche dont les propos ne sont pas reliés au champ logique mais à celui de la linguistique visant la délimitation des deux catégories - syntagmes nominaux désignatifs et termes d'adresse - et la description de leur fonctionnement. Le problème de la délimitation de ces deux catégories et, implicitement, celui de la constitution d'un appareil terminologique couvrant les catégories en question, se reflète dans un article de Delphine Perret (1968) qui oppose les vocatifs - « termes par lesquels on s'adresse à quelqu'un » au terme d'adresse désignatif "par lequel on mentionne quelqu'un dans son discours » (Perret, $1968: 3$ ). Il est étonnant que l'auteur parle de « terme d'adresse désignatif » dans la mesure où le mot «adresse » renvoie automatiquement à la

${ }^{1}$ Comme l'expliquent les auteurs du Dictionnaire de linguistique et des sciences du langage (1995: 45), « on appelle fonction appellative, la fonction grammaticale remplie par les appellatifs de la communication directe. Cette interpellation de l'interlocuteur par le locuteur est traduite par le vocatif dans les langues casuelles $»$.

${ }^{2}$ Dans cette perspective, nous voudrions souligner l'importance des travaux d'A. Zwicky qui pose la question du vocatif en anglais moderne, F. Braun (1988) et C. Kerbrat-Orecchioni (1992). 
fonction vocative, tandis que le mot «désignatif» est clairement du côté de la mention. On peut supposer que cette difficulté terminologique est conditionnée par le problème de savoir comment concilier ces deux catégories. La définition proposée par D. Perret comporte un autre problème : celui de la juxtaposition de terme d'adresse et de mention, puisque l'un ne peut être équivalent à l'autre. Toutefois, on soulignera que l'auteur de l'article "Termes d'adresse et injures » pose les deux notions comme opposés, non comme identiques, et associe le terme d'adresse vocatif à la deuxième personne de l'énonciation - points importants que nous allons retenir pour notre étude.

Dans le premier chapitre de sa thèse consacrée aux «Désignatifs et termes d'adresse dans quelques textes en moyen français », D. Lagorgette définit ces deux catégories dans l'usage linguistique et dans la description grammaticale. Elle propose de distinguer les termes d'adresse des désignatifs et caractérise ces derniers de la manière suivante :

Nous nommerons désignatifs des syntagmes nominaux présents dans le récit et le discours. Ces syntagmes renvoient aux personnages et permettent de les identifier. Ils peuvent contenir indifféremment des noms propres ou des noms communs ainsi que des articles, des pronoms possessifs ou démonstratifs, des adjectifs et/ou des adverbes reliés par la conjonction de coordination et. Ces groupes nominaux peuvent occuper la fonction de sujet ou de complément d'objet dans la phrase. La classe des désignatifs ne renvoie donc pas à une catégorie grammaticale précise. (Lagorgette, $1998: 85$ )

Nous adopterons le choix terminologique de l'auteur et retenons comme noyau de cette définition que les désignatifs sont des syntagmes nominaux de dénomination et de désignation des personnages qui sont employés dans le cadre de la mention des locuteurs et font partie du réseau de référence dans le récit et le discours, peuvent prendre diverses formes, renvoient indifféremment aux trois personnes de l'énonciation et remplissent les fonctions de sujet ou d'objet. En effet, résumer le fonctionnement des désignatifs, cela revient à dire qu'ils sont la pierre de touche du réseau de référence. Conformément aux fonctions syntaxiques occupées, ils se trouvent agents de l'action ou objet du prédicat. On ne peut en dire autant des termes d'adresse qui sont des syntagmes nominaux détachés, et n'ont pas de ce fait, de fonction syntaxique dans la phrase.

Une mise au point terminologique s'impose également pour les termes d'adresse. En effet, dans la littérature linguistique des années 70 et 80 on constate un remplacement du terme de "vocatif», faisant référence aux langues à déclinaison, par celui d' «appellatif». B. Cerquiglini évoque très fréquemment l'appellatif dans son premier chapitre de La parole médiévale (1981). Selon l'étude de D. Lagorgette, le terme d'appellatif dont use B. Cerquiglini est en fait à « traduire » par terme d'adresse. La prise en considération des textes représentatifs de la tradition grammaticale consacrée aux notions "vocatif», " apostrophe » et «appellatif», permet de constater que l'on opère souvent une relation de type métonymique entre la fonction remplie par ces syntagmes et leur nature même. Certes, le système morphologique des langues à déclinaison est caractérisé par l'existence d'un cas vocatif, marqué par des terminaisons spécifiques et utilisé pour 
l'adresse à un allocutaire. Mais cette utilisation spécifique du cas vocatif nous invite plutôt à parler de la fonction vocative. Le groupe nominal qui remplit cette fonction et que les grammairiens nomment vocatif par commodité, sera appelé terme d'adresse (Lagorgette, 1998 : 87). Il doit être distingué de cet aspect fonctionnel qu'implique le terme de « vocatif ».

La description de la relation à établir entre les notions apostrophe et terme d'adresse révèle le même phénomène: assimilation de la fonction syntaxique remplie (apostrophe) et de l'élément syntaxique qui remplit cette fonction (terme d'adresse). L'aspect fonctionnel se trouve au centre de la définition proposée par les auteurs de la Grammaire d'aujourd'hui pour la notion d'apostrophe :

L'apostrophe est exclusivement liée au mode d'énonciation discursive (...). Elle consiste en effet à nommer, par l'une de ses désignations possibles, le destinataire, singulier ou collectif, à qui on adresse la parole en vue d'attirer (ou de retenir) son attention. L'apostrophe relève donc de la fonction phatique du langage. (M. Arrivé, F. Gadet, M. Galmiche, $1986: 67-68$ )

Dans la mesure où l'opération d'interpellation nommée apostrophe peut s'appliquer à des noms de choses personnifiées, nous nous interrogeons sur le statut de l'allocutaire : le terme en apostrophe renvoie-t-il à un être humain ou non ? $\mathrm{Ne}$ serait-il pas envisageable de distinguer les termes d'adresses, réservés aux êtres humains, de l'apostrophe personnifiée, renvoyant aux non-humains dans leur ensemble ? L'intégration de ces réflexions dans le cadre théorique tracé par D. Maingueneau (1994) nous permet de trouver des éléments de réponse :

Les personnes ne peuvent à priori être que des sujets parlants (ou au moins capables de compréhension linguistique). A cela on pourrait objecter qu'il est bien des exemples, pas nécessairement littéraires, d'énonciateurs s'adressant à des êtres inanimés. En réalité, il faut bien comprendre que parler à des individus ou des objets non-parlants revient à les constituer en sujets linguistiques à travers son énonciation : s'adresser à un arbre ou au Destin, c'est en faire ipso facto des individus doués des prérogatives de la personne. Rien de tel pour la non-personne, qui concerne n'importe quels référents, humains ou non, animés ou non, concrets ou non (Didier, la beauté, une table, ...). (Maingueneau, $1994: 22$ )

En effet, c'est à travers l'énonciation qu'un poète comme Charles d'Orléans (1394-1465) «constitue en sujets linguistiques» les abstractions personnifiées Beauté, Danger, Vieillesse, Amour ... qui deviennent des allocutaires capables d'interpréter la parole et « doués des prérogatives de la personne ».

Nous dirons donc qu'il existe une fonction syntaxique, le vocatif, l'appellation ou l'apostrophe, remplie par des syntagmes nominaux que l'on nommera termes d'adresse. Les termes d'adresse sont des syntagmes nominaux détachés sans fonction dans la proposition. Cette catégorie se distingue par sa morphosyntaxe, du fait de son extrême mobilité dans les énoncés et aussi du fait de sa totale indépendance : un énoncé dont on supprime le terme d'adresse ne perd en rien sa grammaticalité. Quant à la nature des éléments assumant la fonction vocative, il reste à préciser qu'ils sont composés de pronoms personnels, de noms propres ou de noms communs, parfois développés par des expansions. Le mot d' "adresse » renvoie donc à la fonction vocative. Les groupes nominaux appelés termes 
d'adresse visent donc un allocutaire de la deuxième personne dans le discours direct. Le fait de placer le terme d'adresse dans le cadre du discours direct et donc de l'interaction verbale donne à cette approche une perspective pragmatique.

Les premières études des termes d'adresse ont été effectuées par les ethnologues et les anthropologues, dans le cadre de descriptions de systèmes sociaux et linguistiques. Les termes d'adresse proprement dits n'y sont que des éléments constitutifs de tout un ensemble de rituels d'échanges interpersonnels qui peut inclure des éléments autres que les syntagmes nominaux.

Depuis les travaux des sociologues (R. Linton, 1947 ; E. Goffmann, 1972) et des sociolinguistes américains (R. Brown et A. Gilman, 1960; R. Brown et M. Ford, 1961) puis anglais (P. Brown et S. Levinson, 1978 et 1987), l'usage des termes d'adresse dans les interactions verbales est nettement caractérisé par rapport au statut social du locuteur et ses variations, d'une part, et par rapport aux rituels sociaux de hiérarchie et de respect, d'autre part. En effet, les termes d'adresse permettent de repérer les interactions entre personnages ainsi que les variations de statut. Lorsque l'on interpelle quelqu'un, on (re)définit la relation que l'on entretient avec cette personne. Le choix d'un terme d'adresse est le résultat d'une opération de sélection parmi les différents termes d'adresse qui sont à la disposition du locuteur selon sa classe, sa culture et la relation qui lie les interlocuteurs. L'adresse opère, comme le remarque Pierre Le Goffic (1993: 374) à la fois une identification (désignation) et une qualification (caractérisation). Comme nous allons le montrer tout au long de notre étude, les termes d'adresse renvoient à différentes facettes caractéristiques des personnages car ils permettent de mettre en relief les traits pertinents pour l'interaction entre les locuteurs dans le domaine des relations sociales.

Avant d'étudier le reflet de cette interaction dans les différents textes de notre corpus regroupant des textes des périodes de l'ancien et du moyen français et donc de prendre en considération la perspective pragmatique, appliquons tout d'abord une perspective formelle visant la description des paramètres selon lesquels la parole s'inscrit dans un texte qui fut écrit en vers, puis mis en prose. Cette démarche s'impose pour un corpus de textes constitué d'éléments «traditionnels 》- des textes en vers - et de témoignages d'une forme d'écriture nouvelle, la prose, qui se crée au XIIIe siècle.

\section{Du vers à la prose : le «destin » des termes d'adresse.}

B. Cerquiglini (1981) a montré selon quels systèmes fonctionne la démarcation du discours dans des textes rédigés d'abord en vers et ensuite mis en prose. C'est en s'appuyant sur les divers textes du Joseph d'Arimathie ${ }^{3}$ que l'auteur de La

${ }^{3}$ A la fin du XIIe siècle, Robert de Boron donne une version chrétienne et globalisante des légendes du Graal introduites par Chrétien de Troyes. De la trilogie romanesque en vers écrite par Robert, seul le premier texte nous est parvenu, qui raconte l'histoire de Joseph d'Arimathie. Cette 
parole médiévale étudie l'étendue, la diversité et la redondance des marques qui signalent la prise de parole dans les textes en vers et en prose. Le récit qui intègre une parole doit donner à son sujet une double information : qu'il y a discours, tout d'abord, et les deux coordonnées de ce discours (le locuteur et l'allocutaire). Pour les textes rédigés en vers, la prise de la parole est signalée majoritairement par une séquence linguistique précédant le discours et comportant un verbe déclaratif: «nous dirons qu'il y a dans ce cas localisation du discours en prolepse » (Cerquiglini, 1981:22). La prolepse n'est que très rarement un début absolu. En effet, le verbe de déclaration est souvent rattaché à une série de verbes qui le précèdent: Quant Joseph Vaspasyen vist, Contre lui se lieve et li dist : (Roman de l'Estoire dou Graal, vv.2035-2036).

Le discours est souvent non seulement annoncé par un verbe déclaratif, mais, par compensation, signalé explicitement en son début. L'utilisation des termes d'adresse s'inscrit dans ce contexte. On part souvent du principe que les termes d'adresse sont simplement des moyens de flécher le discours vers un allocutaire, et par là même redondants, ou facultatifs. Comment expliquer alors les forts taux de fréquence des termes d'adresse dans les textes de notre corpus? Comment expliquer que deux locuteurs en tête-à-tête se gratifient de termes d'adresse ? Ils ne peuvent douter de l'identité de la personne à qui ils parlent, et savent qu'elle sait que leur discours lui est adressé. Toutefois, la notion d'étiquette apposée sur l'allocutaire par le locuteur est un fil conducteur qui nous ramène au problème plus vaste de la dénomination, et aux relations entretenues par le locuteur avec les noms qu'on lui attribue dans le cadre social. L'universalité du phénomène de l'adresse à elle seule suffit à souligner combien l'optique selon laquelle la seule fonction des termes d'adresse serait de pointer l'allocutaire ne peut expliquer la subtilité et la variété des systèmes mis en œuvre. Le pouvoir informatif en revanche fournit une première explication bien plus satisfaisante, que nous suivrons comme une piste dans notre étude. En effet, comme l'a souligné B. Cerquiglini, «la puissance informative de l'appellatif est certainement la cause de sa très grande fréquence dans les textes » (1981:24). L'information fournie par le terme d'adresse - pour reprendre notre terminologie - est double : signaler le début du discours et indiquer l'allocutaire. Mais, dans la plupart des cas, la double information livrée par le terme d'adresse est redondante, comme dans l'extrait suivant du Roman de l'Estoire du Graal : Au tierz jour ha a Joseph dist : "Joseph, or m'enten un petit (...)» (vv. 3443-3444).

Selon ce type de fonctionnement, le terme d'adresse se transforme en relais affirmant le début d'un discours dont les coordonnées figurent dans le contexte (prolepse et contexte plus vaste permettant d'identifier le référent d'un allocutaire intégrant la prolepse sous forme pronominale).

œuvre qui va alimenter les grands cycles romanesques du XIIIe siècle, est au début du siècle mise en prose. 
La prise de la parole peut également être signalée rétrospectivement, en analepse. Le discours est donc désigné comme ayant commencé: "Nous ne savons, ce li disoient, (...)» (Roman de l'Estoire dou Graal, v. 1378). Dans cette construction, aucune marque n'annonce le début du discours. Le recours à des éléments fonctionnant comme marque (et devenant ainsi des marqueurs de la présence d'un discours direct), remédie à cette situation. Nous utiliserons la notion de marque au sens de «borne » ou «signal » servant à distinguer une prise de parole. Rappelons que dans les productions écrites du Moyen Âge, l'absence de marques typographiques (guillemets, italiques, majuscules) est compensée par un marquage lexical très régulier. Les termes d'adresse s'avèrent comme étant particulièrement rentables dans la mesure où ils apportent, comme informations nouvelles, non seulement l'identité de l'allocutaire, mais la présence même du discours. L'analepse devient un moyen de mise en valeur de la présence d'un discours qui n'a été annoncé que de façon faible ou vague.

Quand la parole naît au sein du récit, les moyens utilisés pour la signaler se décrivent sous forme d'oppositions : prolepse ou analepse ; présence ou absence de dire dans la prolepse ; présence ou absence, en tête du discours, d'un élément de relais : adverbe ou terme d'adresse, etc. Quand la parole succède à une autre parole, déjà en place, le nombre, la diversité des moyens de signalisation s'accroissent, leur nature même change: le système de signalisation ne peut plus se saturer par dichotomie (prolepse ou analepse ; présence de dire ou d'un verbe au sémantisme plus large, ...), mais par énumération. Les marques deviennent de plus en plus fines; tout se passe comme s'il y avait moins d'information à transmettre. Même si la prolepse reste un moyen de signalisation fiable au sein du dialogue, il faut dire que, dans les systèmes en vers, une grande partie de l'information est donnée, comme par inertie, par le discours précédent. La parole présuppose une réponse, une nouvelle prise de parole qui fera du locuteur présent l'allocutaire, par croisement. Ceci ne dispense cependant pas de marques qui signalent qu'un discours attendu prend effectivement place; elles désignent où ce discours commence. Ces marques, davantage bornes que signalisation, sont de nature diverse. Dans la catégorie des marques lexicales, catégorie constituée des impératifs et des termes d'adresse, ce sont surtout les groupes nominaux appelés termes d'adresse qui sont particulièrement fréquents. Dans son étude consacrée au récit de Robert de Boron, B. Cerquiglini (1981 : 32-33) signale également la présence de marques rhétoriques (divers procédés de reprise qui placent en tête du discours un élément emprunté au discours précédent) et de marques prosodiques (la parole a pour clôture le couplet qui constitue le cadre de la parole et qui fonctionne comme un moule prosodique).

Dans la mesure où la prose abandonne ce qui lui paraît relever de l'ajout prosodique, les marques lexicales devraient donc jouer un rôle primordial dans le processus de signalisation de discours qui caractérise le passage du vers à la prose. En effet, les termes d'adresse et les interjections sont en général maintenus par la prose :

«-Sire, dist Joseph, granz merciz!

Je demant le cors de Jhesu. » 
(Roman de l'Estoire dou Graal, vv.454-455)

Et Joseph respont : « Sire, grans mercis, et je demant le cors de le prophete. » (version en prose, ms E, 129-130)

Comme la comparaison de ces deux passages nous permet de le constater, la prose extrait la séquence linguistique en incise pour l'antéposer. On notera qu'un et d'allocution (Cerquiglini, 1981: 41) apparaît après le groupe terme d'adresse + interjection au début de ce qui est proprement le contenu du discours. Il représente une sorte de seconde attaque, une seconde prise de parole mettant en valeur la puissance informative de la séquence qui suit et qui, comme la combinaison terme d'adresse + interjection, « est également du discours ».

La plupart des discours du texte versifié, une fois repérés, sont fortement explicités et instanciés par la prose, mis en prolepse. «Les discours non introduits, en vers, rentrent dans le moule commun des discours proleptiques, ils passent du vide au plein » (Cerquiglini, 1981: 47), de sorte que chaque type de discours du texte versifié reçoit le complément nécessaire pour le faire adhérer à un modèle quasi unique. Les informations contenues dans le discours ont, étant donné la présence de la prolepse, un faible rendement. Il en est ainsi du terme d'adresse. La prose, afin de constituer la séquence proleptique, extrapose le terme d'adresse du discours en vers; celui-ci devient l'objet indirect du verbe de déclaration, selon le schéma suivant: vers $《 \mathrm{~A}$, dist $\mathrm{B}, \ldots.\rangle \rightarrow$ prose $\mathrm{B}$ dist a $\mathrm{A}: \ll \ldots$. .

L'allocutaire est désigné avant le discours: si le terme d'adresse est maintenu par la prose, il redouble cet allocutaire et n'a qu'une fonction de relais. La prose peut également faire apparaître un terme d'adresse qui n'était pas exprimé en vers. Mais que ce terme d'adresse soit maintenu ou apporté, il importe de voir qu'il est toujours redondant : il redouble l'allocutaire donné dans la prolepse. Dans le cadre de la présente étude nous nous interrogeons sur la portée de cette "plénitude structurelle » (Cerquiglini, 1981: 53) : n'est-elle caractéristique que du phénomène de mise en prose de modèles versifiés ou, au contraire, s'agit-il d'une spécificité de toutes les productions textuelles en prose (indépendamment de la présence d'un texte modèle en vers) à la fin du Moyen Age ?

Avant la mise en relation des termes d'adresse relevés dans notre corpus constitué de textes en ancien et en moyen français, avec les séquences proleptiques, nous nous intéresserons, dans un premier temps, à la nature ainsi qu'à la forme des termes d'adresse dans une perspective de comparaison avec les réalités sociales.

\section{Analyse du Corpus.}

\subsection{Le monde en vers et en prose.}

Le reflet des réalités sociales dépend, comme nous allons le montrer, moins de la forme du texte que du regard de l'auteur sur la réalité qui l'entoure. Ce regard est conditionné par le statut de l'auteur, son rôle dans la société ainsi que les univers littéraires à construire. Nous ne rencontrons pas dans chaque texte l'image complète de la hiérarchie sociale dont la complexité dépasse souvent le cadre étroit du 
schéma trifonctionnel reconnu par Georges Dumézil comme une structure fondamentale de la culture indo-européenne. Dans leur article Idéal social et vocabulaire des statuts, J. Batany et J. Rony (1971) montrent que les conditions contraignantes de l'épopée médiévale, par exemple, impliquent l'exploitation de certains registres lexicaux. Etant donné que l'une des finalités de l'épopée est la mise en valeur de la catégorie sociale des chevaliers - l'un des éléments constitutifs du schéma trifonctionnel - dans son rôle de protecteur de la société, il est normal de trouver dans les chansons de geste un réseau sémio-lexical des catégories lexicales dans lequel les autres catégories ont un rôle secondaire de contrepoint.

La Chanson de Roland est caractérisée par l'omniprésence de «ceux qui combattent » et donc de l'ordre des guerriers qui n'était pas non plus homogène. On pourrait y distinguer trois groupes. Il y avait d'abord une haute noblesse issue de lignées princières dominant une province entière, et qui, sans pour autant renier sa subordination par rapport au souverain, se substituait entièrement à lui dans le gouvernement de son domaine. Un second ensemble était celui des vassi dominici, les compagnons du roi (comites, d'où comtes), constituant originellement le groupe de ses proches et de ses fidèles, son escorte, une bande de compagnons d'armes liés à lui par un engagement d'homme à homme. Loin au-dessous de cette aristocratie était le groupe beaucoup plus nombreux des chevaliers ; d'origine souvent modeste, c'étaient de véritables soudards, des guerriers pillards et violents.

Les termes d'adresse visant à titre de référent une collectivité - celle des chrétiens face à celle des païens - regroupent pour le monde des chrétiens les représentants des deux premières catégories de l'ordre des guerriers: Seignurs, Baron(s), Seignurs barons, Franceis, Barons franceis, Francs, Chevaler, Francs chevalers. Le locuteur appartenant au monde chrétien pointe son allocutaire appartenant à ce même monde par le choix d'un terme d'adresse mettant en valeur sa position sociale (seigneur, baron), son rôle de combattant à cheval (chevalier) et son appartenance ethnique (franc, français). Il est à noter que les discours circulant à l'intérieur du monde païen sont caractérisés par les mêmes syntagmes nominaux à fonction vocative: Seignurs, Seignurs baruns Barun(s), Franc chevalier vaillant auxquels s'ajoute le terme d'adresse païen (5occurrences) ${ }^{4}$. Ainsi, au vers 2844 , Baligant, le chef suprême des païens, s'adresse à ses guerriers en utilisant ce terme : «Venez, paien, car ja s'en fuient Franc». Dès que les deux interlocuteurs représentent respectivement ces deux univers opposés, le vocabulaire de la stratification sociale est remplacé par des qualificatifs dépréciatifs : Glutun, Culvert, Culvert païen, Feluns Français.

Ce qui vaut pour les collectivités, vaut également pour leurs meilleurs représentants. Quant à Charlemagne, le Chanson de Roland nous dépeint un portrait

${ }^{4}$ Les Sarrasins du Roland comme des autres chansons de geste, sont pour l'essentiel une création littéraire; leur religion en particulier est encore plus éloignée de l'islam que du christianisme (Bancourt, 1982, 341-493 et Daniel, 2001, 133-194). Aussi vaut-il mieux éviter de les désigner comme musulmans et utiliser, comme le texte, les termes de sarrasins et de païens, parler de l'algalife et non du calife, et appeler Baligant amiral plutôt qu'émir. 
de roi féodal en même temps qu'élu de Dieu. Les termes reis et emperere relevés en adresse, témoignent de sa position statutairement supérieure. Ces deux termes fonctionnent en général comme le noyau nominal d'une structure expansive : dreiz emperere, reis magnes, bels sire reis, sire emperere mais également reis orguillos quand les locuteurs sont deux messagers païens. Le contenu social du terme d'adresse sire, quand il est utilisé seul (sept occurrences pour le référent Charlemagne), est réduit à un minimum. Ce terme, tout en assurant la fonction de marque de début de discours, pointe l'allocutaire sans le nommer, accomplit le geste vide de l'identification. Son fonctionnement répond à celui d'un terme minimum (Cerquiglini, 1981 : 26). La valeur sémantique de base du terme sire qui implique l'expression d'une idée de différence hiérarchique, l'exercice d'une autorité, ressort dans les syntagmes nominaux sire reis ou sire emperere dont le premier terme désigne la supériorité hiérarchique et le second la fonction exercée.

La société dans laquelle vivent les Sarrasins se modèle à peu près complètement sur la société chrétienne. Il s'agit d'une société féodale, dans laquelle le roi est aussi chef militaire. Rien d'étonnant alors si les fidèles de Marsile et de Baligant, l'incarnation totale de la menace païenne, s'adressent à leurs maîtres en les appelant reis (gentilz reis, sire reis). La seule note d'exotisme est apportée par certains noms empruntés à l'arabe, mais utilisés sans véritable rapport avec leur valeur étymologique. A la tête du monde païen se trouve un amiral (d'amir, émir, « chef »), dont la position évoque plutôt celle d'un calife. Nous relevons deux emplois du terme amiralz en adresse. Dans ces deux attestations, amiralz n'est jamais utilisé seul, mais s'appuie toujours sur un autre terme (Sire amiralz, v.2790) ou un syntagme nominal (Sire reis, amiralz, v. 2831). Tout se passe alors comme si le contenu social de ce terme « exotique » ne pouvait être explicité que par une mise en relation avec les structures sociales dans le monde chrétien. Il est à noter que le titre algalife que porte l'oncle de Marsile n'est jamais utilisé en adresse.

L'une des images qu'on retient traditionnellement de la Chanson de Roland est celle de l'empereur entouré de ses douze pairs, et de l'évident parallèle ainsi établi avec le Christ et ses apôtres. Qui sont les douze pairs ? A l'origine, les comtes du palais, comites palatini, ceux qui étaient chargés du service personnel du roi, ses fidèles, les vassi dominici dont il a été question dans le cadre de la présentation de l'ordre des guerriers. Dans la tradition épique, il y avait douze pairs parce que chacun était censé servir l'empereur un mois par an avec ses troupes, mais aussi pour le parallèle évangélique déjà mentionné. Leur liste varie selon les textes. Ils se regroupent presque tous, dans le Roland, par couples de compagnons, dont le plus célèbre est celui formé par Roland et Olivier. L'importance du compagnonnage épique se reflète aussi dans les termes d'adresse. En effet, le trait essentiel qui caractérise Olivier est d'être le compagnon de Roland. Dans leurs rapports mutuels, le vocabulaire de la stratification sociale est dominé par celui servant à exprimer la relation de compagnons d'armes : cumpainz/ sire cumpainz/ sire cumpainz, amis/ Olivier, frere / Olivier, compaign, frere / bels cumpainz Olivier.

On peut mettre aux côtés de Roland et Olivier un troisième personnage qui formerait alors avec ces deux compagnons d'armes un trio de combattants bien individualisés: l'archevêque Turpin. Son statut d'ecclésiastique permet de le 
caractériser comme étant un représentant de «ceux qui prient ». Il n'en figure pas moins parmi les meilleurs chevaliers francs et les plus hardis. L'idéal que Turpin représente est celui d'une Eglise soumise avant tout au pouvoir royal. Il combat en priorité au titre de vassal de l'empereur, comme en témoignent les termes d'adresse relevés dans le texte et qui ne font aucunement allusion à son statut d'ecclésiastique : Sire/ Gentilz hom / Gentilz hom, chevalier de bon aire.

L'épopée est un monde d'hommes, et le compagnonnage est la forme que prend la tendresse (ami, frere) dans un tel monde. La tendresse au féminin s'incarne dans le nom d'Aude, mais aucun terme d'adresse faisant référence à ma gente sorur Alde (Olivier, v.1720) n'est attesté dans le texte. Le seul terme d'adresse renvoyant à une femme est celui de dame (v.2724) utilisé par le messager de l'amiral pour s'adresser à la reine Bramimonde, femme de Marsile. Celle-ci se substitue naturellement au roi, son mari, trop faible pour recevoir les visiteurs. Bramimonde ce faisant se comporte exactement comme n'importe quelle reine chrétienne de chanson de geste et mérite donc le même terme d'adresse (même s'il s'agit d'un terme minimum, équivalent du masculin sire) qu'une grande dame de l'univers chrétien.

Un autre univers poétique intéressant du point de vue des termes d'adresse à contenu social est celui de Rutebeuf, poète parisien du XIIIe siècle, poète des realia (Payen, 1984). La prise en considération des questions politiques et religieuses de son temps (les croisades, les ordres mendiants, la querelle des universités) ainsi que l'évolution des structures sociales (expansion économique, rôle des villes, éveil industriel et développement intellectuel) expliquent la nature ainsi que la forme des termes d'adresse relevés dans les Euvres Complètes. Ce sont surtout les représentants de l'aristocratie et les prélats qui se trouvent au centre de l'intérêt de notre poète. Les représentants de l'état ecclésiastique sont soit individualisés - les termes remplissant une fonction vocative intègrent des syntagmes nominaux du type adjectif qualificatif + terme à contenu social (fols chanoines, « Le miracle du sacristain », v.249) ou nom commun (terme de respect) + nom commun à contenu social (dan prestres, c'est-à-dire monsieur le prêtre, «La dame qui fit trois fois le tour de l'église », v.116) -, soit traités en tant que communauté (prelat, clergie). La perspective de généralisation qui caractérise ces emplois au pluriel est quelque peu neutralisée grâce à l'intégration de ces collectifs dans des structures expansives avec un/des adjectif(s) qualificatif(s) (prelat esnervoié "prélats sans énergie »; Clerc aaise et bien sejournei, Bien vestu et bien conraei «clercs vivant dans le confort et le repos, bien vêtus et bien pourvus ») ou des compléments prépositionnels (prelat de saint esglise; Prelat auz palefrois norrois " prélats aux palefrois norvégiens $»)$. Les éléments constitutifs du vocabulaire de la stratification sociale désignant à titre de référent les représentants de l'aristocratie sont traités de la même façon: à titre individuel dans des syntagmes nominaux de type titre + complément désignant la terre d'origine (cuens de Blois), adjectif qualificatif + titre + terre d'origine (Gentilz cuens de Poitiers), titre honorifique + nom propre + terre d'origine (Messire Erars de Valeri), titre ou fonction + nom propre + structure appositive exprimant le respect du locuteur ou un lien de parenté (cuens Jehan, biau tres dolz sire, /Rois Hanrris, freres au bon roi,...), en tant que collectivité regroupée dans un pluriel généralisant (Baron, qu'aveiz vos enpancei?, "La nouvelle 
complainte d'outre-mer», v.103) ou présentée sous un aspect fonctionnel particulier (chevaliers de plaiz et d'axises «chevaliers qui siégez aux tribunaux », Tournoieur).

A la complexification des structures sociales répondent dans la poésie de Rutebeuf des termes d'adresse de plus en plus complexes, c'est-à-dire se présentant sous forme de structures de coordination et de séries énumératives, regroupant les différents représentants d'une même catégorie sociale (Vous devin et vous discretistre, ... « vous, théologiens, et vous, juristes $» / /$ Empereor et roi et conte et duc et prince, ...//Et vos (roi) et li cuens de Poitiers, Et li autre baron ensemble... // prince, baron, tournoieour Et vos autre sejorneour, ... « princes, barons, amateurs de tournois, et vous autres, les paresseux »), ou rassemblant plusieurs couches sociales (Prelat, clerc, chevalier, borjois qui..., Dites // Oeiz, prelat et prince et roi, ...).

Cette volonté consistant à introduire dans son univers poétique les reflets de la réalité sociale et surtout de la vie quotidienne continue à se manifester chez les poètes de la période du moyen français, et, en particulier, chez Eustache Deschamps (1344-1404). L'œuvre de Deschamps est un dialogue avec la société, celle de ses maîtres et de ses amis. Ses textes nous transmettent le point de vue d'un personnage issu d'une couche subalterne de la noblesse, appartenant à la cour à plein titre, mais dans une situation de dépendance permanente et tirant ses revenus de son insertion dans l'administration monarchique. La cour représente dans la production de Deschamps une sorte de microcosme exemplaire, à la fois positif et négatif. L'habitant de ce microcosme est désigné par le terme générique curial que Deschamps utilise également en adresse sans expansion ou avec une relative explicative (Vous, curiaulx, que juenesse demaine, $\mathrm{n}^{\circ} 1296$, t.VII, p.48-49). Comme chez Rutebeuf, les termes d'adresse relevés visent un ou plusieurs personnages individualisés cités nommément (Hugues d'Ars, Garences, Rossoy, Prunelé et vous, Florigni, ..., n ${ }^{\circ} 803$, t.IV, v.1-2) ou une collectivité désignée sous forme d'un pluriel généralisant accompagné ou non d'un qualifiant (Gens d'armes; Dames; Seigneurs; Gens de l'Eglise; peuple plain d'orgueil) ou d'une périphrase constituée d'un pronom personnel et d'une relative déterminative (Vous qui a court royal servez ; Vous qui la guerre menez,...).

L'expérience faite par Eustache Deschamps dans l'administration monarchique explique le grand nombre de termes spécifiques désignant les fonctionnaires de l'appareil administratif du roi (baillif, prevost, secretaire, gouverneur, seneschal, maire, conseillier, juge, notaires). Quelques-uns de ces termes apparaissent également en adresse accompagnés d'une proposition subordonnée relative qui spécifie la nature de la fonction exercée (Juges qui les loys gardez, ... ; Notaires qui instrumens fais, ...).

Les termes d'adresse prenant la forme d'une structure de coordination ( $\mathrm{a}$ et $\mathrm{b} ; \mathrm{a}, \mathrm{b}$ et c) concernent exclusivement les représentants de la haute aristocratie (Empereurs, Roys, et tous princes de terre, Avisez bien vostre gouvernement, ...; Prince et tout Roy qui gouvernement $a, \ldots$ « Poésies morales et historiques », p.148; Prince, Seigneur, et toute poesté De royaume, de pays, de cité, Qui gouvernez pour mieulx garder défence, ... p.4). La troisième strophe de la ballade $n^{\circ} 1475$ est constituée de deux séries impressionnantes de termes d'adresse dont la seconde se distingue de la première par 
l'emploi de formes au singulier ainsi que par la présence du coordonnant et qui annonce le dernier élément de cette série de termes :

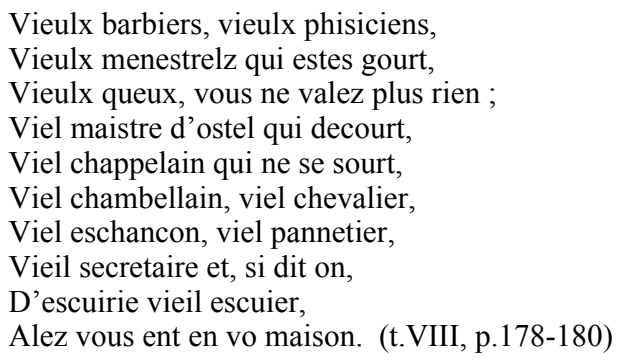

Les vieux serviteurs de la cour sont sacrifiés sur l'autel de la Jeunesse. Pour montrer qu'aucun office, du ménestrel au chambellan, n'échappe à ce sort, Deschamps s'adresse dans la dernière strophe de cette ballade aux représentants de quelques métiers de l'Hôtel.

Le milieu de la cour reste le milieu privilégié d'un autre auteur de la période du moyen français: Charles d'Orléans (1394-1465). L'univers allégorique de ses ballades est peuplé par toute une troupe d'abstractions personnifiées qui vivent sous le règne d'un monarque particulier: le Dieu d'Amour. En dehors des termes Amour(s) et Dieu d'Amours qui sont attestés en tant que désignatifs comme en tant que termes d'adresse et qui mettent en valeur la nature particulière du souverain du royaume d'Amours, les autres termes choisis par les habitants de l'univers allégorique pour s'adresser à leur maître, témoignent de la volonté du poète de créer un univers poétique aussi vrai que le monde réel : Sire, Prince, Vostre Seigneurie, Vostre Excellence, Roi. Tandis que le terme d'adresse Prince lorsqu'il renvoie à Amour, est toujours accompagné d'adjectifs qualificatifs (Noble Prince; Treshault et noble puissant Prince; Tresexcellent, treshault et noble Prince) qui confirment le sème social («le premier dans une hiérarchie ») présent dans Prince, le terme d'adresse Sire qui est spécialisée dans la fonction vocative, fonctionne comme un terme minimum. Aucune expansion par un adjectif qualificatif n'est attestée. Pourtant des combinaisons du terme Sire avec des adjectifs comme beaus, douz, chiers, gentils ou riches étaient très fréquentes au Moyen Age. Dans la poésie amoureuse de Charles d'Orléans, la fonction de dénomination du terme d'adresse Sire tend à zéro ; sa fonction première consiste à indiquer le début du discours.

Dans un autre texte représentatif de la littérature médiévale, les emplois du terme Sire concernant les possibilités d'association à un adjectif qualificatif ou la combinaison avec un déterminant possessif sont plus diversifiés. Mais, comme chez Charles d'Orléans, la vision du monde est strictement aristocratique. Avec ce texte nous quittons le « monde en vers" afin d'entrer dans celui en prose : La Mort le Roi Artu (1230). Dans ce texte se définit d'emblée un univers de référence en rapport avec les plus hautes sphères politiques, les pauvres n'ont aucune existence sociale 
réelle. L'auteur, se réfugiant dans l'imaginaire, se crispe sur une vision strictement aristocratique du monde, comme pour réagir à l'installation des bourgeois dans les rouages du pouvoir ${ }^{5}$. Ce repli sur la féodalité, sur les valeurs de la haute aristocratie se reflète également au niveau des termes d'adresse. Le terme Sire est omniprésent et, dans tous les cas, le référent est un représentant de l'aristocratie (le roi Arthur : 142 occurrences, Lancelot, Gauvain, Yvain, Hector, Bohort ainsi que Mordret et Agravain), ce qui confirme que la valeur sémantique de base de ce terme implique l'expression d'une idée de différence hiérarchique, du respect et de l'autorité. Sire est donc employé pour s'adresser aux rois ou aux grands seigneurs féodaux ainsi qu'aux représentants de l'état ecclésiastique. Le titre honorifique messire (missire) a un caractère exclusif dans la mesure où il n'est attribué qu'aux meilleurs chevaliers (Lancelot, Gauvain, Yvain) ainsi qu'aux grands seigneurs féodaux (le roi Yon); dans toutes les attestations le terme messire est suivi d'un nom propre de personne. Dans la combinaison Sire chevaliers, terme de respect choisi, par exemple, par la demoiselle d'Escalot pour s'adresser à Gauvain, Sire fonctionne comme un terme générique, marqueur d'une position sociale élevée, tandis que chevaliers précise l'appartenance à la catégorie de « ceux qui combattent». Les adjectifs qualificatifs qui se combinent avec le terme d'adresse Sire sont biaus et douz (Lancelot à l'un des frères d'Escalot: Biaus sire; la reine à Lancelot: Biaus douz sire). Ces combinaisons témoignent plutôt d'un comportement respectueux que d'une distinction sociale. Dans les termes d'adresse Rois Artus et mestre(s), c'est l'aspect fonctionnel qui est mis en valeur : la fonction du roi et celle du médecin (mire de Lancelot). Tandis que le terme d'adresse Rois est toujours accompagné du nom propre Artus (" formule officielle»), mestre, en dehors de deux emplois autonomes, s'associe aux mêmes adjectifs qualificatifs que Sire : biaus et douz, qui atténuent la coloration sociale/ fonctionnelle du terme d'adresse.

En ce qui concerne les personnages féminins, nous notons le nombre important d'occurrences (70) du terme d'adresse Dame presque exclusivement ${ }^{6}$ réservé à la reine Guenièvre. Nous relevons une seule structure expansive, structure dont la complexité semble répondre à la difficulté de la situation vécue par la reine : Dame debonere seur toutes autres dames et plus cortoise que nule autre (paragraphe 92 : le peuple s'adresse à la reine lorsqu'elle est conduite au bûcher). Mais dans la majorité des attestations le terme d'adresse Dame se comporte comme le terme minimum Sire et devient donc son équivalent féminin.

Le royaume arthurien est un monde qui se dérègle de l'intérieur. La lutte des clans touche dans La Mort Artu à son paroxysme. L'appartenance à tel ou tel clan et donc la précision des rapports de parenté devient un paramètre important pour la

\footnotetext{
${ }^{5}$ Philippe Auguste favorise, au détriment de la noblesse, l'essor politique et économique d'une bourgeoisie que justement La Mort Artu ignore.

${ }^{6}$ Dans deux attestations, le terme d'adresse Dame renvoie à la tante des deux frères d'Escalot. Il est à noter que dans ces deux attestations le terme a été choisi par l'un des deux neveux. En effet, la famille des frères d'Escalot appartient au monde des nobles mais n'occupe que le rang inférieur dans la pyramide féodale (le père n'est qu'un vavasseur, un arrière-vassal).
} 
caractérisation des personnages. Le vocabulaire de la parenté et des liens familiaux entre donc en concurrence avec celui de la stratification sociale. Les termes d'adresse mettant en valeur la relation de parenté qui lie les interlocuteurs, se présentent dans le texte sous forme de syntagmes nominaux composés d'un adjectif qualificatif laudatif et d'un terme de parenté (biaus niés, biax oncles, biax cousins, biaus/douz frere, bele suer) en emploi autonome ou en tant que structure appositive qui suit un nom propre de personne (Boort, biaus cousins ; Agravain, biaus freres, ...).

La position sociale et le lien de parenté avec l'interlocuteur déterminent également le contenu et la forme des termes d'adresse dans un autre texte en prose, caractéristique de la période du moyen français et représentant le monument essentiel de 1'histoire en français les Chroniques de Jean Froissart'. L'image que nous propose Froissart de la société du XIVe siècle est dominée par un climat idéologique tout à la fois royal, aristocratique et chevaleresque. Lorsque Jean Froissart annonce qu'il va rappeler les hauts faits chevaleresques de son temps, il n'entend pas faire l'éloge aveugle de la chevalerie, mais faire apparaître les troubles de l'époque et le sens de l'histoire à travers les péripéties de l'institution elle-même la plus chargée de sens grâce à la littérature. L'univers de la chevalerie est omniprésent comme en témoignent les termes d'adresse. A titre de terme de base pour le fonctionnement en adresse nous relevons, comme dans les autres textes, le terme minimum Sire, utilisé pour les représentants de la haute aristocratie (rois, comte de Hainaut, duc de Normandie...). Sire peut devenir le noyau nominal d'une structure expansive comportant des adjectifs qualificatifs (chiers sire, gentilz sire) ou un complément prépositionnel précisant la terre d'origine d'un grand seigneur de la féodalité (Sire de Maubuisson). Dans ce dernier type d'emploi, le terme Sire commute avec un terme désignant un titre de noblesse précis : contes de Haynau, contes de Montfort. L'emploi des titres honorifiques messire et monseigneur est intéressant dans la mesure où monseigneur semble être réservé aux rois (anglais et français) ainsi qu'aux membres de la famille royale (Louis d'Espagne s'adresse à Charlon de Blois, neveu de Philippe VI, en l'appelant «Monsigneur», §177). L'attribution des titres de messire et de monsigneur à un même référent ( $\$ 262$ Monsigneur Thumas, §281 Messires Thumas) témoigne d'une certaine hésitation dans le processus de généralisation des formes de cas régime pour la fonction vocative, processus qui se croise d'ailleurs, dans cet exemple précis, avec une spécialisation des emplois des deux formes de singulier.

Dans la plupart des attestations, monsigneur apparaît en emploi autonome, c'està-dire sans aucun complément ou qualifiant ${ }^{8}$, ce qui n'est pas le cas de la forme messire, ancienne forme de cas sujet, qui est toujours suivie d'un nom propre de personne, en l'occurrence le prénom d'un chevalier de renommée (Messire Gautier, Messire Jehan, Messire Agos). A côté de ces emplois au singulier, nous relevons

\footnotetext{
${ }^{7}$ Nous avons étudié les termes d'adresse du Premier Livre (achevé en 1373).

8 Nous relevons cependant deux exceptions: Monsigneur Guillaume, chiers amis (§40) et Monsigneur Thumas (\$262).
} 
également des termes d'adresse au pluriel renvoyant à des groupes/ensembles de chevaliers. Le collectif disponible pour ce type d'emploi est extrait du même paradigme ; il s'agit de l'ancienne forme du cas sujet pluriel : signeur, qui s'insère dans des structures du type nom + nom (Signeur chevalier, Signeur compagnon) ou adjectif + nom (chier signeur, biau signeur). Toujours dans le domaine des collectifs, le Premier Livre des Chroniques contient deux occurrences du nom féminin gent, précédé de l'adjectif qualificatif bonne, en adresse : Bonne gent,... (\$237, d'Arteveld s'adresse aux Gantois), Ma bonne gent, ... (\$263, le roi français s'adresse au peuple de Paris). Avec le terme gent nous quittons l'univers de la chevalerie afin de nous rapprocher de la population des villes (les bourgeois, le peuple). Le terme bourgeois n'est pas attesté en adresse ${ }^{9}$, mais il est intéressant de constater que les bourgeois qui sont cités nommément dans le texte méritent le même terme d'adresse que les représentants de l'aristocratie: Messire Ustasse (\$320, le roi Edouard III s'adresse à l'un des six bourgeois de Calais). Il s'agit évidemment des bourgeois les plus riches et les plus influents de la ville dont les mérites valent bien une ascension sociale.

Avec les termes d'adresse au féminin nous retournons dans l'univers aristocratique. En effet, ce n'est que le terme Dame choisi pour les représentantes de la haute aristocratie (la sœur du roi de France, la reine d'Angleterre, la comtesse de Salisbury) qui remplit une fonction vocative dans le texte.

Ce sont les femmes qui se trouvent au centre d'un texte rédigé par un auteur dont la grande affaire est la défense et l'illustration des femmes : Christine de Pizan. Les hommes du Moyen Âge ont longtemps conçu la femme comme une catégorie ; mais ce n'est que tardivement qu'ils y ont fait intervenir distinctions sociales et activités professionnelles pour nuancer les modèles de comportement qu'ils lui proposaient. Avant d'être paysanne, châtelaine ou sainte, « la» femme s'est trouvée définie par son corps, son sexe, et ses relations avec des groupes familiaux. Épouse, veuve ou vierge, c'est par rapport à un homme ou à un groupe d'hommes qu'on en a dessiné la personnalité juridique et l'éthique quotidienne ${ }^{10}$. Une partie importante de l'œuvre de Christine de Pizan, première femme de lettres professionnelle de la littérature française, s'inscrit dans le cadre d'un effort didactique répondant aux préoccupations propres à cet auteur, celles qui sont déterminées par sa condition et par son sexe. L'éloge des femmes trouve son expression la plus forte dans la Cité des dames (1404/1405) et dans le Livre des trois vertus (ou Trésor de la cité des dames, 1405) qui indique aux femmes leurs devoirs selon leur état. S'adressant aux princesses, puis aux femmes de noble état, enfin à celles de condition modeste, Christine prodigue, selon le modèle tripartite qui lui est cher, des conseils de toute

\footnotetext{
${ }^{9}$ Le Premier Livre contient des emplois de « bourgeois » en tant que désignatif, surtout au paragraphe 312 («Les six bourgeois de Calais»: ....se leva en piés li plus riches bourgeois de la ville, que on clamoit sire Ustasse de Saint Pière, ... / uns aultres très honnestes bourgois et de grant afaire ...)

${ }^{10}$ Pour une analyse approfondie du statut de la femme dans la société médiévale: J. Le Goff, L'homme médiéval, éd. du Seuil, 1989 ; R. Delort, La vie au Moyen Âge, éd. du Seuil, 1982 ; G. Duby, Le chevalier, la femme et le prêtre. Le mariage dans la France féodale. Paris, Hachette, 1981.
} 
nature. La première partie concerne les «princesses et haultes dames ». Les termes d'adresse relevés dans cette partie se concentrent dans une lettre rédigée par la gouvernante à l'intention de sa maitresse. A titre de terme de base nous relevons celui de Dame actualisé par le possessif ma marquant la relation de subordination personnelle, ainsi que des adjectifs qualificatifs traduisant les notions de respect (redoubtee) et de politesse (chiere). Une structure de coordination associe le terme spécifique princepce (aspect fonctionnel) à celui de Dame, plus générique (marqueur de catégorie sociale): Tres redoubtee princepce et ma chiere dame, ... (I, 281). Dans la seconde partie de son ouvrage, Christine s'adresse aux «dames, damoiselles et femmes de court ou service des princepces et haultes dames ». Dans la mesure où nous restons dans la catégorie des nobles (petite noblesse), le terme de dame est toujours utilisé en adresse mais commute, à l'intérieur de cette deuxième partie, avec celui de femme qui acquiert une coloration sociale grâce à l'ajout de compléments déterminatifs (femmes de court ou service des princepces). Le terme d'adresse Dame disparaît complètement de la troisième partie consacrée aux « femmes d'estat, bourgoises de citez et bonnes villes ». Le terme générique femme $\mathrm{y}$ est omniprésent et intègre des structures expansives qui permettent d'établir des différences sociales évidentes entre les femmes qui n'appartiennent pas au milieu de la cour. En effet, pour les expansions à l'aide d'adjectif qualificatifs nous relevons d'un côté riches femmes et de l'autre miserables femmes ou bonnes povres femmes. L'expansion peut se manifester aussi sous forme de compléments de nom signalant l'appartenance au modèle hiérarchique (femmes d'estat) ou à un milieu social/géographique précis (femmes de bonnes villes), ainsi que sous forme de propositions subordonnées relatives caractérisant ces femmes du point de vue de leur activité (vous qui servez / femmes qui portez le nom de crestianté et qui le convertissez en si vil office, ...).

Comme nous avons pu le constater tout au long de notre étude, le monde en vers - dans ses grandes structures - ne diffère pas de celui en prose. Les syntagmes nominaux détachés assumant une fonction vocative permettent, en ce qui concerne le référent visé, de reconnaître les représentants du schéma trifonctionnel. Les auteurs imposent leur vision (aristocratique, générale, féminine) du monde, ce qui explique pourquoi on ne trouve pas dans chaque texte l'image complète de la hiérarchie sociale. La complexification de la société s'accompagne de l'apparition d'un vocabulaire de plus en plus spécialisé mettant en valeur l'image d'une société plus que trifonctionnelle, comme en témoignent l'univers poétique de Rutebeuf et la poésie du quotidien d'Eustache Deschamps. Mais face à ce mouvement de renouveau lexical qui prend note des modifications dans la réalité sociale, nous notons une très forte tendance à la conservation de termes d'adresse de base comme Sire/Seigneur ou Dame qui traversent tous les textes, indépendamment de la vision de l'auteur, et apparaissent dans des structures expansives relativement figées (Chiers Sire, Biaus (dolz) Sire; Belle Dame, Chiere Dame). Le maintien de la forme n'exclut aucunement une évolution des valeurs d'emploi. Un bel exemple de cette évolution se manifestant sous forme d'une transformation du sens («transferred meaning », Stowell, 1908: 201), est l'emploi du terme d'adresse Sire, précédé du qualificatif beau, dans le roman Jehan de Saintré (1456). Le 
décalage avec les modèles romanesques traditionnels qui caractérise ce roman d'Antoine de la Sale, transparaît également au niveau des termes d'adresse. Beau sire est le terme d'adresse que choisissent Belle Cousine et ses dames pour se moquer de l'innocence de Saintré ou dans l'intention de flatter celui-ci. Ces transformations socio-sémantiques sont l'indice de l'évolution des structures sociales mais aussi, comme dans Saintré, des réévaluations et des réajustements auxquels la chevalerie se soumet.

Après avoir étudié les termes d'adresse dans une perspective de comparaison avec les réalités sociales, nous nous intéressons dans la dernière partie de notre travail à la place ainsi qu'au fonctionnement du terme d'adresse en tant que signal pour la présence d'un discours.

\subsection{La localisation du discours.}

Quand la parole naît au sein du récit, les moyens qu'utilisent les auteurs de textes en vers ou en prose pour la signaler se décrivent avant tout sous forme de l'opposition prolepse ou analepse. Mais la localisation en prolepse ou analepse ne dispense pas de marques qui renforcent ce système : celles-ci mettent en valeur le fait qu'un discours attendu prend effectivement place; elles désignent où ce discours commence. Comme nous l'avons déjà souligné, parmi les marques lexicales, ce sont les termes d'adresse qui sont particulièrement fréquents. Ils remplissent une double fonction : signaler, tel un adverbe, une interjection, le début du discours ; indiquer l'allocutaire. La puissance informative des termes d'adresse est certainement la cause de leur très grande fréquence dans les textes. Dans la Chanson de Roland, par exemple, nous relevons 129 termes d'adresse qui signalent le début d'un discours attendu et localisé préalablement par une prolepse. Dans la majorité des emplois ( 87 occurrences), le terme d'adresse se trouve en position initiale absolue, tandis que dans 42 discours directs localisés par une séquence proleptique il n'apparaît qu'après un autre élément avec lequel il se partage la fonction de signalisation de début de discours (interjection, impératif, exclamation). L'utilisation d'un terme d'adresse en position finale n'est attestée que pour quinze discours pour lesquels il faut cependant apporter une précision. En effet, pour treize discours position finale signifie que le terme remplissant la fonction vocative se place à la fin du premier d'une série plus ou moins longue d'énoncés constituant le discours d'un personnage ${ }^{11}$. La distance entre la séquence proleptique et le terme d'adresse qui font partie d'un dispositif commun, est donc réduite à un minimum. Pour les deux occurrences restantes, le terme d'adresse clôt le discours en même

\footnotetext{
${ }^{11}$ E cil respunt : " Morz estes, Baligant! Ja vostre deu ne vos erent guarant. Carles est fiers e si hume vaillant: Unc ne vi gent ki si fust cumbatant. Mais reclamez les barons d'Occiant, Turcs e Enfruns, Arabiz e Jaianz. Ço que estre en deit, ne l'alez demurant.» (vv.3513-3519) (Celuici répond: «Vous êtes mort, Baligant! Jamais vos dieux ne vous protégeront. Charlemagne est intrépide, et ses hommes vaillants ; je n'ai jamais vu de peuple aussi combatif. Faites donc appel aux barons d'Occïant : Turcs et Enfruns, Arabes et Géants. Quoi qu'il arrive, ne tardez surtout pas!»
} 
temps que la laisse dans laquelle il figure. Il est à noter que dans ces deux attestations le discours ne se réduit qu'à un seul énoncé et la distance séparant le terme d'adresse de la prolepse reste donc minimale. Mais indépendamment de leur place à l'intérieur du discours, ces 87 occurrences de termes d'adresse s'appuient sur une séquence annonciatrice qui précède le discours. Mais le système du vers a également recours à un système de signalisation qui fonctionne rétrospectivement et désigne le discours comme ayant commence: l'analepse. Les termes d'adresse trouvent dans l'analepse leur domaine majeur. Ils apportent dans ce cas, comme informations nouvelles, non seulement l'identité de l'allocutaire, mais la présence même du discours. Dans la Chanson de Roland les analepses prennent la forme exclusive d'incises (29 attestations); des emplois en fin d'énoncé ne sont pas attestés. Vingt des vingt-neuf discours signalés rétrospectivement débutent, en effet, par ce signal fort qu'est le terme d'adresse (dans deux cas associé à un impératif), suivi de l'incise. Dans 99\% des incises nous relevons le verbe déclaratif minimal dire qui s'impose donc largement devant son seul et unique concurrent: le verbe respondre (un emploi ; v.1026). L'identité de l'allocutaire étant fournie grâce au terme d'adresse, la plupart des incises ne mentionne pas l'allocutaire. L'accent est donc mis sur le locuteur qui intègre l'incise sous forme d'un nom propre, d'un syntagme nominal ou pronom personnel. Mais dans six incises, l'information de la présence d'un discours - véhiculée par le verbe dire - est complétée par les deux coordonnées de ce discours : le locuteur et l'allocutaire. Dans quatre de ces six incises sur-informées, l'allocutaire est présent sous forme pronominale, substitut de l'allocutaire désigné nommément par le terme d'adresse ("Bels filz Malprimes, Balgant li ad dit, ... », v.3201). A partir du moment où le terme d'adresse se présente sous forme d'un terme minimum (sire) dont le référent est identifié avant le discours, c'est l'incise qui se chargera, à travers l'utilisation d'éléments du paradigme désignationnel du terme en question, d'une nouvelle mise en relation du terme d'adresse minimum avec son référent ( Jo vos aim mult, sire, dist ele al cunte,...», v.635). Sept des vingt-neuf incises débutent par le démonstratif neutre ço, qui désigne la partie de discours déjà prononcée en explicitant la nature proprement métadiscursive de la construction en analepse: intervention d'une parole qui en identifie une autre.

Mais retournons maintenant du côté des séquences proleptiques et comparons la structure et la puissance informative de celles-ci avec les incises. Il est à souligner que le verbe déclaratif minimal dire, dont la fonction unique est de signaler le discours (en introduisant le locuteur et éventuellement l'allocutaire), l'emporte toujours (55 occurrences) sur les autres verbes disponibles pour remplir cette même fonction (respondre : 18 emplois ; crier/escrier : 13 emplois ; apeler : 12 emplois ; parler : 5 emplois; reclamer : 4 emplois ; demander : 2 emplois ; commander : 1 emploi). Ces verbes au sémantisme plus large annoncent un discours, mais s'appuient plus fortement sur d'autres marques qui, par compensation, signalent explicitement le début de ce discours. Un signal particulièrement rentable est le terme d'adresse : Li empereres en apelet Rollant: " Bel sire niés, ..., v.783 / Et cil respundent : "Sire, ..., v.946 / Li emperere recleimet ses Franceis : "Seignors barons,.. », v.3405. Les verbes de parole peuvent être coordonnés (Guenes $i$ vint, 
..., Par grant veisdie cumencet a parler, E dist al rei, vv.674-676) ou rattachés à une série de verbes d'action qui les précèdent (Olivier... Fiert l'Algalife..., Trenchet la teste, Brandist sun colp...., E dist après, vv.1952-1958).

Quant à l'indication de l'allocutaire dans la prolepse, l'auteur fournit cette information sous forme d'un pronom personnel, d'un syntagme nominal ou d'un nom propre. Cette présence de l'allocutaire dans la prolepse, n'affaiblit-elle pas la puissance informative (double fonction : début de discours + allocutaire) du terme d'adresse ? En effet, dans la plupart des cas, la double information livrée par le terme d'adresse est redondante. Pour les prolepses comportant un allocutaire pronominal, le terme d'adresse n'est redondant que dans la mesure où il relaie, en clair, une information donnée plus haut: il reprend en fait un allocutaire pronominal, substitut lui-même de l'allocutaire désigné nommément dans l'entourage (Vers Sarrazins reguardet fierement $E$ vers Franceis humele $e$ dulcement; Si lur ad dit un mot curteisement : Seignurs barons, ... vv.1162-1165). La redondance est dans ce cas minimale, mais elle existe. La construction exemplaire est donc celle où allocutaire et terme d'adresse sont nominaux. Dans son étude consacrée à la mise en prose de l'histoire de Joseph d'Arimathie, B. Cerquiglini (1981: 24) a relevé dans le texte en vers un cas de redondance flagrante : Au tierz jour ha a Joseph dist : "Joseph, or m'enten un petit ... » (R, 3443-44). Ce type d'exemple n'est pas attesté dans la Chanson de Roland, mais nous y relevons dix cas de redondance avec le verbe déclaratif minimal dire (1 cas pour le verbe parler, 12 cas de redondance pour le verbe apeler et 4 pour reclamer $^{12}$ ). Malgré la redondance, il est à souligner que la présence du terme d'adresse permet de traiter le référent visé selon deux aspects différents, comme par exemple l'appartenance à un même clan familial et la place dans la hiérarchie féodale (Dist à sun uncle belement en riant : Bel sire reis, ... ", vv.862-863). Le désignatif figurant dans la prolepse considère le référent comme élément constitutif d'un groupe / d'un ensemble familial, ethnique ou fonctionnel, tandis que le terme d'adresse se charge de l'expression du statut social.

La prise en considération de la position du terme d'adresse et de la relation à établir entre le terme à fonction vocative et la séquence localisatrice nous a permis de caractériser ces termes comme étant des signaux particulièrement clairs et dotés d'une réelle puissance informative. Vérifions maintenant si cette caractéristique est aussi valable pour le système de la prose. En effet, aussi bien dans la Mort Artu que dans les Chroniques de Froissart ou dans le roman Jehan de Saintré, la position privilégiée des termes d'adresse est la position initiale absolue, ce qui justifie la comparaison du terme d'adresse avec le guillemet ouvrant. Dans la Mort Artu, par exemple, le terme d'adresse sire est attesté aussi bien en position initiale absolue ou

${ }^{12}$ Pour les verbes apeler et reclamer, la question de la redondance ne se pose pas de la même façon dans la mesure où ces deux verbes commandent un complément d'objet direct désignant l'allocutaire. Dans toutes les attestations celui-ci intègre la prolepse sous forme nominale. Le discours qui suit débute par un terme d'adresse qui fonctionne un peu comme le guillemet ouvrant du Moyen Age. 
au début du discours après une interjection ou un adverbe d'assertion, qu'en position médiane (séparant ainsi la principale d'une subordonnée complétive) ou finale absolue. Mais la répartition des emplois est plus que déséquilibrée : 218 occurrences de ce terme en position initiale absolue (auxquelles s'ajoutent pour l'ouverture du discours 13 emplois après un adverbe d'assertion et dix après une interjection), contre une seule utilisation en position finale absolue. Ces chiffres témoignent d'ailleurs du fonctionnement spécifique du terme d'adresse sire, le « premier degré de l'appellatif » (B. Cerquiglini, 1981:26), qui tout en assurant la fonction de marque de début de discours, pointe l'allocutaire sans le nommer. Si en effet l'allocutaire est suggéré par la cohérence contextuelle, si le nommer est répétitif, il est possible de désigner ce dernier par un terme minimum. Ce type de fonctionnement ainsi que la tendance générale consistant à placer le terme d'adresse, et surtout le terme minimum sire, au début du discours se confirme dans les Chroniques ainsi que dans le roman du XVe siècle Jehan de Saintré. En ce qui concerne ce dernier texte, nous notons quand même une légère augmentation du nombre de termes d'adresse en position finale ( 25 attestations; ce qui reste évidemment peu face aux 449 attestations à l'ouverture du discours). Ces vingt-cinq attestations se concentrent surtout dans des répliques - réponses de dialogues qui ne sont pas localisées par une prolepse ou analepse ${ }^{13}$.

L'articulation proleptique reste le mode privilégié de localisation d'un discours dans le système de la prose. Il y a cependant quelques nuances à apporter. En effet, dans la Mort le Roi Artu, nous relevons 250 séquences proleptiques renforcées par le signal terme d'adresse. Le nombre des incises s'appuyant sur un terme d'adresse qui précède cette séquence localisatrice se rapproche "dangereusement» du nombre relevé pour les prolepses (234). La présence simultanée d'une séquence localisatrice et d'un terme d'adresse ainsi que la puissance informative qui se cache derrière cet ensemble (« il y a du discours »), neutralise quelque peu l'importance du placement des éléments. Le déséquilibre traditionnel se réinstalle quand on s'intéresse au nombre des analepses - un emploi attesté dans le roman La Mort le Roi Artu- ainsi qu'aux modes de localisation d'un discours utilisant un terme d'adresse dans les Chroniques (166 prolepses, 24 incises et 2 analepses) et dans Jehan de Saintré (283 localisations en prolepse, 104 incises et 2 analepses). Ces données nous permettent d'en déduire que l'ordre privilégié est: séquence localisatrice + terme d'adresse. Si le terme d'adresse précède la séquence qui localise le discours, il représente soit l'élément d'ouverture unique du discours, soit se combine avec une autre marque signalant la présence d'un discours (interjection, adverbe d'assertion) ou un début d'énoncé (question, énoncé déclaratif) complété après l'incise.

En ce qui concerne les verbes de parole utilisés dans les séquences qui signalent la présence d'un discours, on constate une relative pauvreté lexicale. Dans La Mort

13 -Et combien vous a elle envoyé ? - Soixante escuz, Madame. - Soixante escus ? dist Madame. Vous en avez la moittié cabassé ! - Non ay, par ma foy, Madame. (p.134) 
le Roi Artu nous relevons pour les articulations proleptiques majoritairement le déclaratif minimal dire ainsi que respondre, demander, crier, escrier / soi escrier. Le seul verbe possible en incise est le verbe faire, toujours au présent et toujours avec inversion du sujet. Dans les Chroniques, l'incise ne fonctionne qu'avec les verbes dire et respondre, tandis qu'Antoine de la Sale dans son roman Jehan de Saintré utilise exclusivement le verbe dire dans les incises localisant un discours comportant un terme d'adresse. Etant donné cette omniprésence du verbe déclaratif minimal dire, faut-il en déduire que le terme d'adresse et son entourage lexical sont assez puissants pour préciser les circonstances et la nature du dire? En effet, le système de la prose associe très souvent le verbe dire de la prolepse à un autre verbe de parole ou un verbe d'action / de mouvement permettant de préciser les circonstances de l'acte de parole et de préparer la tonalité du discours mis en place après la séquence proleptique (Mort Artu: tent son gage et dit au roi / la reine fist trop grant duel et li dist// Chroniques : ...li respondi et li dist / se getta en genoulz et dist ensi // J. de Saintré : en toucha la main et dist / la remercia et dist / a Messire Enguerran s'enclina et dist).

Terminons notre étude par quelques remarques concernant la question de la redondance du terme d'adresse. Dans La Mort le Roi Artu, par exemple, le redoublement d'un allocutaire présent sous forme pronominale dans la prolepse, est un procédé largement exploité ( 27 attestations du modèle «si dist» face à 96 emplois de « (si) li dist » avec ou sans verbe coordonné). En effet, il ne s'agit là que d'une redondance grammaticale. Même pour les allocutaires nominaux, la redondance n'est jamais flagrante (même terme pour l'emploi en tant que désignatif et en tant que terme d'adresse). Pour les noms communs désignant l'allocutaire dans la prolepse, la reprise s'effectue en général à l'aide d'un terme d'adresse minimum (Lors dit au roi : "Sire, ... ", 85/9); tandis que les noms propres sont majoritairement repris sous forme d'un terme d'adresse rappelant un rapport de parenté (Et lors dit a Morgain : "Bele suer, ... », 52/14). De même dans Jehan de Saintré, le nombre des séquences proleptiques comportant un allocutaire pronominal est supérieur à celui des prolepses sans indication de l'allocutaire (pour le verbe déclaratif minimal dire : 140 emplois du type «il li dist» contre $30 \mathrm{du}$ modèle « il dist »). Quant aux allocutaires nominaux, les désignatifs utilisés dans la prolepse se transforment majoritairement en terme d'adresse minimum (...dist bellement a la royne: "Madame, .... », p.182 / Messire Enguerran dist au roy: "Seigneur, ... », p.208). Nous relevons cependant quelques cas de redondance plus marquée qui se manifestent essentiellement dans des situations de communication qui réunissent plus que deux interlocuteurs. Cette «sur-information» résulte de la volonté de pointer l'allocutaire, de donner une nouvelle direction à la communication ou, d'une façon beaucoup plus banale, de marquer la plupart des discours par l'ajout d'un élément caractéristique : les termes d'adresse. L'écriture n'est pas une vocation chez Antoine de La Sale, encore moins une profession. Le monde des lettres lui est assez étranger. Il en témoigne une certaine maladresse, comme par exemple dans l'extrait suivant où le terme choisi pour identifier le locuteur et le terme d'adresse qui vise l'allocutaire sont identiques : « A ! Madame, 
dist Madame a la royne, vous tailliez larges corroyes d'aultrui cuir.» (p.184). La précision de l'identité de l'allocutaire dans l'incise s'impose donc.

Ce récit, qui mêle non sans ambiguïté le ton du roman de chevalerie à celui du fabliau, se nourrit largement des discours directs : discours d'éducation mondaine et chevaleresque, leçons de morale, rencontres amoureuses.

Comme nous avons pu le constater pour tous les textes de notre corpus, les termes d'adresses sont des signaux particulièrement forts permettant d'identifier la mise en place d'un discours, indépendamment de son contenu. Leur puissance informative est certainement la cause de leur très grande fréquence dans les textes. N'oublions pas que les discours non localisés par une séquence spécifique s'appuient essentiellement sur des marques lexicales (termes d'adresse, impératifs) ou rhétoriques qui mettent en valeur la présence d'un discours.

L'emploi des termes d'adresse dans les textes étudiés obéit finalement à deux contraintes : marquer le début du discours et préciser l'identité de l'allocutaire. La séquence localisatrice précède majoritairement le terme d'adresse. Ceci est valable pour le système du vers ainsi que pour celui de la prose. La rencontre d'un terme d'adresse et d'un allocutaire identifié dans la séquence localisatrice pose le problème de la redondance du terme d'adresse qui, comme nous avons le montré, n'en est pas vraiment un dans la mesure où la redondance n'est que grammaticale lorsqu'il s'agit d'un allocutaire pronominal. Et même pour les allocutaires nominaux, nous sommes, dans la majorité des attestations, loin d'une impression de sur-information, telle qu'elle se dégage de l'analyse de B. Cerquiglini pour les textes mis en prose à partir de modèles en vers. Au contraire, la reprise d'un désignatif sous forme d'un terme d'adresse aboutit majoritairement à l'emploi d'un terme minimum dont la fonction première est la signalisation du début du discours. Le renforcement de la fonction de signalisation d'un début de discours va de pair avec l'affaiblissement de l'expression d'un contenu social précis. Madame et sire visent un référent qui appartient à la haute noblesse, mais l'identité sociale précise ne ressort qu'à travers la prise en considération des informations fournies par le contexte.

La mise en relation des termes d'adresse avec les réalités sociales nous a permis de constater que la prépondérance d'une catégorie sociale dans un texte ne correspond pas toujours au rôle réel de cette catégorie dans la société. C'est la vision de l'auteur, son propre statut social, l'objectif de l'acte d'écriture, le genre textuel qui conditionnent la répartition des formes ainsi que la présence ou l'absence d'un type social.

\section{RÉFÉRENCES BIBLIOGRAPHIQUES}

Corpus

BORON, R. de (1927) : Le Roman de l'Estoire dou Graal. Edition critique par W.A. Nitze, Paris, Champion.

La Chanson de Roland (2003) : Edition critique par Cesare Segre, Genève, Droz. 
DESCHAMPS, E. (1878-1903) : Euvres complètes. Paris, Firmin-Didot.

FROISSART, J. (2001): Chroniques. Livres I et II. Editions et textes présentés et commentés par P.F. Ainsworth et G.T. Diller, Paris, Librairie Générale Française (Lettres Gothiques).

La Mort le Roi Artu (1996) : Edition critique par J. Frappier, Genève, Droz.

ORLEANS, Ch. de (1992) : Ballades et Rondeaux. Paris, Librairie Générale Française (Lettres Gothiques).

PISAN, Ch. de (1989) : Le livre des trois vertus. Paris, Champion.

RUTEBEUF (2002): Cuvres complètes. Texte établi, traduit, annoté par M. Zink, Paris, Librairie Générale Française (Lettres Gothiques).

SALE, A. de la (1995) : Jehan de Saintré. Edition et présentation de J. Blanchard, Paris, Librairie Générale Française (Lettres Gothiques).

Ouvrages consultés

ALLETON, V. (1981) : "Les termes d'adresse en chinois contemporain », in Diogène, 116, 46-75, Paris, Gallimard.

ARRIVÉ, M., GADET, F., GALMICHE, M. (1986) : La grammaire d'aujourd'hui, Paris, Flammarion.

BANCOURT, P. (1982): Les musulmans dans les chansons de geste du cycle du roi. Aix-en-Provence, Publications de l'université de Provence.

BATANY, J. (1970) : " Paradigmes lexicaux et structures littéraires au Moyen Age », in Revue d'histoire littéraire de la France, 5-6, 819-836.

BATANY, J. (1992): Approches langagières de la société médiévale. Caen, Paradigme.

BATANY, J., \& RONY, J. (1971) : «Idéal social et vocabulaire des statuts : Le couronnement de Louis », in Langue Française, 9, 110-118.

BRAUN, F.(1988): Termes of Address. Problems of patterns and usage in various languages and cultures. Berlin, Mouton de Gruyter.

BRERETON, G. (1958) : « Titres et termes d'adresse dans le Ménagier de Paris », in Romania, 79.

BROWN, R., \& FORD, M. (1961): “Address in american English”, in D. Hymes (ed.), Language in Culture and Society, 234-244. New York, Harper and Row.

BROWN, R., \& GILMAN, A. (1960): "The pronouns of power and solidarity", in Thomas A. Sebeok (ed.), Style in Language, 253-276, Cambridge, Mass. and New York, MIT Press and Wiley.

BROWN, P., \& LEVINSON, S.C. (1978): "Universals in Language Usage: Politeness Phenomena. In E. N. Goody (ed.), Questions and Politeness. Strategies in Social Interaction, 56-324, Cambridge, C.U.P.

BROWN, P., \& LEVINSON, S.C. (1987): Politeness: Some Universals in Language Usage. Cambridge, Cambridge University Press.

CERQUIGLINI, B. (1981): La Parole Médiévale: discours, syntaxe, texte. Paris, Les Editions de Minuit.

CORBLIN, F. (1995) : Les formes de reprise dans le discours. Anaphores et chaînes de référence, Rennes, Presses universitaires de Rennes. 
DANIEL, N. (2001): Héros et Sarrasins. Une interprétation des chansons de geste. Paris, Cerf.

DUBOIS, J., GIACOMO, M., GUESPIN, L., MARCELLESI, Ch., MARCELLESI, J.B., MEVEL, J.-P. (1994) : Dictionnaire de linguistique et des sciences du langage, Paris, Larousse.

DUBY, G. (1978) : Les trois ordres ou l'imaginaire du féodalisme. Paris, Gallimard.

FOULET, L. (1950/1951) : «Sire, Messire », in Romania, 71, 1-48 et Romania, 72, 3177, 324-367 et 479-528.

FREGE, G. (1952): Translations from the Philosophical Writings of Gottlob Frege, ed. By P. Geach and H. Black, Oxford, Blackwell.

GARY-PRIEUR, M.-N.(1994): Grammaire du nom propre, Paris, PUF.

GOFFMAN, E. (1972): Interaction Rituals. Essays in Face to Face Behavior. London, Penguin Press.

HUIZINGA, J (1998) : L'automne du Moyen Age. Paris, Payot.

KÄHÄRA, T. (1997) : « Le nom d'adresse Sire en moyen français », in Actes du VIIIe colloque international sur le moyen français. B. Combettes et $\mathrm{S}$. Monsonégo (Eds.). Paris, Didier.

KERBRAT-ORECCHIONI, C. (1992) : Les interactions verbales (2). Paris, Collin.

KLEIBER, G. (1981): Problèmes de référence: descriptions définies et noms propres, Paris, Klincksieck.

KRIPKE, S. (1980): Naming and Necessity, Oxford, Blackwell.

LAGORGETTE, D. (1998) : Désignatifs et termes d'adresse dans quelques textes en moyen français. Thèse de doctorat non publiée, Université de Paris X, Nanterre.

LAGORGETTE, D. (2004): «Termes d'adresse et verbes de parole en moyen français : approche pragmatique », in Actes $d u$ colloque Ci-Dit, Université libre de Bruxelles, S. Marnette, L. Rosier (eds), Paris, L'Harmattan.

LAGORGETTE, D. (2006) : «Du vocatif à l'apostrophe : problèmes terminologiques et théoriques. Termes d'adresse et détachement en diachronie du français », in L'Information Grammaticale, 109, F. Neveu éd., 38-44.

LALANDE, D. (1995) : Lexique des chroniqueurs français. Paris, Klincksieck.

LE GOFFIC, P. (1993): Grammaire de la phrase française, Paris, Hachette.

LINTON, R. (1947): The Cultural Background of Personality. London, Paul Kegan, Trench, Trubner \& Co Ltd.

LEHMANN, S. (2003): La représentation des rapports sociaux dans l'univers allégorique des ballades de Charles d'Orléans: une analyse discursive. Thèse de doctorat non publiée, Université de Paris X, Nanterre.

MAINGUENEAU, D. (1994) : L'énonciation en linguistique française, Paris, Hachette.

MARCHELLO-NIZIA, Ch. (1997) : La langue française aux XIVe et XVe siècles. Paris, Nathan.

PAYEN, J.-Ch. (1984) : «Le je de Rutebeuf ou les fausses confidences d'un auteur en quête de personnage », in Mélanges Erich Köhler, Heidelberg, 229-240.

PERRET, D. (1968) : «Termes d'adresse et injures », in Cahiers de lexicologie, 12, 314. 
PERRET, M. (1994) : « Façons de dire : les verbes de parole et de communication dans La Mort le Roi Artu ", in La Mort du roi Artur ou le crépuscule de la chevalerie. Etudes recueillies par J. Dufournet, Paris, Champion.

RÉCANATI, F. (1979): La transparence et l'énonciation: pour introduire à la pragmatique, Paris, éd. du Seuil, 1979.

STOWELL, W.A. (1908): Old-French Titles of Respect in Direct Address, Baltimore.

ZWICKY, A. (1974): "Hey, what's your name!, in Chicago Linguistic Society, 10, 787801. 